\title{
Shading and Shadowing of Architecture in Mixed Reality
}

\author{
Tetsuya Kakuta \\ Graduate School of \\ Interdisciplinary \\ Information Studies, \\ The University of Tokyo \\ kakuta@cvl.iis.u-tokyo.ac.jp
}

\author{
Takeshi Oishi \\ Institute of Industrial Science, \\ The University of Tokyo \\ oishi@cvl.iis.u-tokyo.ac.jp
}

\author{
Katsushi Ikeuchi \\ Interfaculty Initiative in \\ Information Studies, \\ The University of Tokyo \\ ki@cvl.iis.u-tokyo.ac.jp
}

\begin{abstract}
We propose a simple method to express shading and shadowing of virtual objects in Mixed Reality especially appropriate for static architecture models in outdoor scenes. We create the shadows of the virtual objects in a fast and efficient way using a set of pre-rendered basis images and shadowing planes. The proposed method is limited in interactivity but can operate in near real-time.
\end{abstract}

\section{Introduction}

For the seamless integration of virtual and real objects in Mixed Reality(MR), it is important to achieve consistency of illumination. There are many previous works related to the consistency of illumination. Jacobs and Loscos provide a detailed survey of illumination methods for MR[5]. They classify the various techniques based on their input requirements of geometry and radiance of real environment[11][4][3][2][1]. Most of these techniques are demonstrated on indoor scenes and few of them are carried out at interactive update rates.

To simulate the naturally illuminated architectural environment, Nimeroff et al. presented an efficient re-rendering method using pre-rendered basis images[6]. Sato et al. applied this technique for MR and achieved a fast image synthesis with natural shading[10]. Nevertheless, their method is applicable only to still images and fixed viewpoints.

\section{The shadowing planes and the basis images}

In this study, we extend Sato et al.'s method for interactive MR application. We generate basis images only to express the shadows of virtual objects and set them onto other planate objects (hereafter called the shadowing planes) so that they correspond to both the arbitrary viewpoints and changing illumination of the real environment. However, our method is applicable only to static models such as architecture.

We generate a set of the basis images at the pre-processing stage. In order to obtain the basis images, we set up the shad-

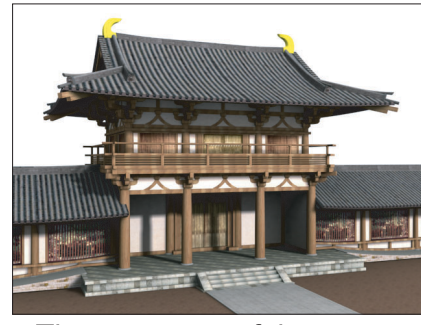

The geometry of the scene

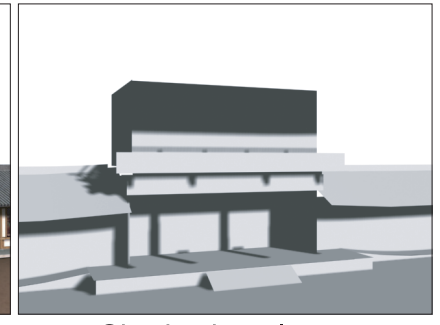

Shadowing planes
Figure 1. The mechanism of shadowing plane.

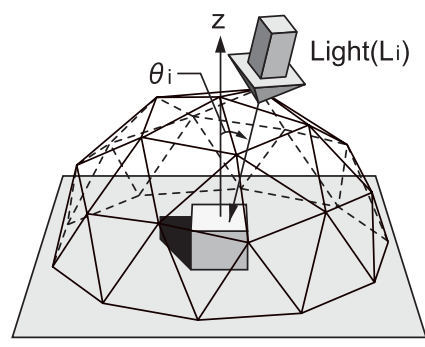

Rendering with directional lights

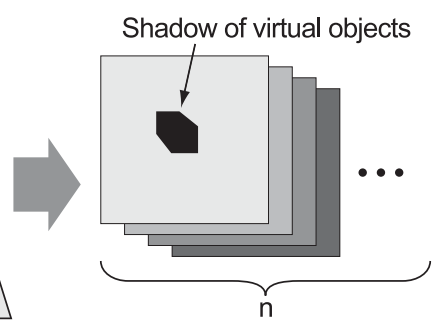

Generated basis images
Figure 2. Generation of the basis images

owing planes manually on the geometry of the scene (fig .1). We previously divided the geometry of virtual and real objects into clusters by apparently organized parts. The shadowing planes were put between these geometric parts and the user's viewpoint. Each shadowing plane records the shadows of virtual objects projected onto itself as the basis images.

After setting up $m$ shadowing planes, we assume that the illumination in outdoor scenes is a hemispheric area light source. Then we approximate it by the assembly of $n$ directional lights $L_{i}(i=1,2, . ., n)$ arranged on a geodesic dome that divides the hemisphere almost evenly. The virtual objects are rendered with each directional light (fig .2). Virtual cameras, which look towards each shadowing plane $P_{j}(j=1,2, \ldots, m)$ perpendicularly, capture the shadows of virtual objects cast on the shadowing planes so that we obtain basis images Ibasis $_{j, i}$ with every shadowing plane.

In the process of synthesis, we capture the omni- 
directional image of the sky and compute luminance parameters $S_{i}(i=1,2, . ., n)$, which show the illumination distribution of the real scene. For shading of virtual objects, we set six virtual directional lights in the scene. The intensity of every light is determined by the parameter $S i$. For the shadowing of virtual objects, we compute the linear combination of the basis images $\operatorname{Ibasis}_{j, i}$ and parameter $S i$ as

$$
\operatorname{Isum}_{j}=\sum_{i=1}^{n} S_{i} \times \text { Ibasis }_{j, i}
$$

where sum $_{j}$ represents the synthesized soft shadow images. Finally we map $I s u m_{j}$, onto every shadowing plane $P_{j}$ as an alpha transparency texture, and we express the pseudo shadows of virtual objects.

\section{Experimental result}

We tested our proposed method in an outdoor scene. Our system is mainly based on Canon's MR Platform system [12]. Fig .3 shows the synthesized images. We can see that both the shading and shadows of a virtual building are reasonably matched to the illumination of the real scene. The omni-directional image of the sky is captured in every frame so that our method can reflect the dynamic change of the illumination.

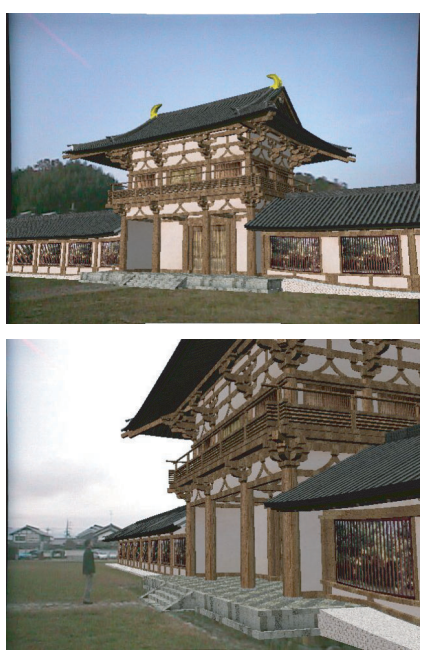

(a) Synthesized image without shadowing
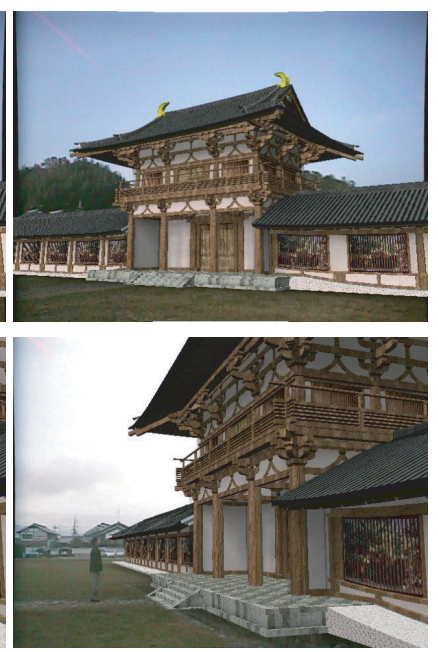

(b) Synthesized image with shadowing
Figure 3. Synthesized image in outdoor scene

\section{Conclusion}

This paper proposes a simple method of shading and shadowing for a static architecture model in MR. The method can apply in outdoor scenes and can yield results in near realtime. However, the shadows of virtual objects are rendered in the preprocessing stage and therefore cannot respond to dynamic alteration of the scene.

\section{Acknowledgement}

This work was, in part, supported by the Ministry of Education, Culture, Sports, Science and Technology, under the program, "Development of High Fidelity Digitization Software for Large-Scale and Intangible Cultural Assets." We are also grateful to the village office of Asuka and the National Research Institute for Cultural Properties. The texture of the CG model in Fig. 1 and Fig .3 is synthesized from the pictures of books[9][7][8].

\section{References}

[1] O. Bimber, A. Grundhofer, G. Wetzstein, and S. Knodel. Consistent illumination within optical see-through augmented environments. In ISMAR '03: Proceedings of the The 2nd IEEE and ACM International Symposium on Mixed and Augmented Reality, page 198, 2003.

[2] P. Debevec. Rendering synthetic objects into real scenes: bridging traditional and image-based graphics with global llumination and high dynamic range photography. In $S I G-$ GRAPH '98: Proceedings of the 25th annual conference on Computer graphics and interactive techniques, pages 189198, 1998.

[3] S. Gibson, J. Cook, T. Howard, and R. Hubbold. Rapid shadow generation in real-world lighting environments. In EGRW '03: Proceedings of the 14th Eurographics workshop on Rendering, pages 219-229, 2003.

[4] M. Haller, S. Drab, and W. Hartmann. A real-time shadow approach for an augmented reality application using shadow volumes. In VRST '03: Proceedings of the ACM symposium on Virtual reality software and technology, pages 56-65, 2003.

[5] K. Jacobs and C. Loscos. Classification of illumination methods for mixed reality. In Eurographics State of the Art Reports, pages 95 - 118, September 2004.

[6] J. S. Nimeroff, E. Simoncelli, and J. Dorsey. Efficient Rerendering of Naturally Illuminated Environments. In Fifth Eurographics Workshop on Rendering, pages 359-373, 1994.

[7] K. Ogawa. Fumetsu no konchiku 1 Horyuji Gojyunoto. Mainichi Shinbunsha, 1988.

[8] K. Ogawa. Fumetsu no konchiku 2 Toshodaiji Kondo. Mainichi Shinbunsha, 1988.

[9] N. rokudaiji taikan kankokai. Nara Rokudaiji Taikan vol.1 Horyuji. Iwanami Shoten, 1972.

[10] I. Sato, Y. Sato, M. Hayashida, F. Kai, Y. Sato, and K. Ikeuchi. Fast image synthesis of virtual objects in a real scene with natural shading. The Institute of Electronics, Information and Communication Engineers, D-2, J84-D-2(8):1234-1242, 2001 (in Japanese).

[11] I. Sato, Y. Sato, and K. Ikeuchi. Acquiring a radiance distribution to superimpose virtual objects onto a real scene. IEEE Transactions on Visualization and Computer Graphics, 5(1):1-12, 1999.

[12] S. Uchiyama, K. Takemoto, K. Satoh, H. Yamamoto, and H. Tamura. Mr platform: A basic body on which mixed reality applications are built. In ISMAR '02: Proceedings of the International Symposium on Mixed and Augmented Reality, page 246, 2002. 